\title{
WOLF-RAYET STAR VARIABILITY AND WIND \\ INHOMOGENEITIES
}

\author{
JOHN C. BROWN and LORNA L. RICHARDSON \\ Department of Physics and Astronomy, University of Glasgow, Glasgow G12 8QQ, \\ Scotland
}

\begin{abstract}
The wind structure diagnostic possibilities offered by combined study of photo, spectro-, and polari-metric variability are discussed using data from the Montréal group. These demand the presence in the wind of localised density inhomogeneities and in particular of large 'blobs' denser than the 'mean' wind and put bounds on the size, mass, and density of individual blobs and their distributions, with implications for theories of blob formation. Blobs responsible for polarimetric variability must be present at the stellar surface rather than forming in the wind, and the relation of this to spectrometric indications that blobs are only detectable at several stellar radii is discussed.
\end{abstract}

Key words: stars: Wolf-Rayet - winds - polarimetry

\section{Introduction}

Broad WR emission line profiles exhibit narrow features in both emission and absorption. Absorption features could be due to thin shells in a spherical wind, but narrow emission features (e.g., Robert 1992; Moffat \& Robert 1992) demand the presence of very localised enhanced emission regions. Their spectral narrowness requires source localisation in the angle $\theta$ their motion makes with the line of sight, while their transience shows their sources to be moving and of small radial extent. On the assumption that the transients, of similar time-scale, in broad-band photometry and polarimetry originate in the same structures, the degree of polarisation and its distinct position angle for each transient show them to be also localised in source azimuthal angle. Such 'blob' data have been studied by Moffat et al. and their physics discussed by Brown et al. (1994). Here we will summarise work in progress on conditions in these blobs, both individually and collectively, from photometric, spectrometric and polarimetric data, both as currently available and with future simultaneous coverage in all three modes.

\section{The optically thin approach}

Analysis of the undoubtedly important radiative transfer effects in WR winds is mainly restricted to steady spherical winds which can say nothing about transient inhomogeneities. In Brown et al. (1994) and the present discussion, analysis of blobs will be in optically thin terms. Horrifyingly naive as this is for RT gurus, it has proved very fruitful in polarimetric modelling of hot star envelopes (Brown \& McLean 1977; Brown et al. 1978; Brown \& 
Wood 1992; Wood et al. 1993) since although predicted fluxes may be in error, their geometric, temporal, and spectral distributions are remarkably accurate and the simple treatment makes the connection between data and model parameters transparent.

\section{Size and density estimates for single blobs}

We consider here how the size and density of a single blob can be inferred from simultaneous observations of its narrow feature flux, spectral width, and its broad-band polarisation. No truly simultaneous data of this kind exist so we will use typical values of each for prominent narrow features (obtained by somewhat hazardous subtraction of a smooth mean profile).

The (diluted) degree $P$ of broad-band polarisation of starlight singly scattered in a localised blob depends only on the total blob electron content $N_{e}=n V$, where $n, V$ are the blob electron density and volume, and on the blob location-distance $r$ from the star's centre and scattering angle $\theta$. If the blob speed is fairly constant and follows the wind speed, $\theta$ is known from the narrow feature shift $\delta \lambda=\Delta \lambda_{\infty} \times \cos \theta$ where $\Delta \lambda_{\infty} / \lambda_{0}=v_{\infty} / c$ with $\lambda_{0}, v_{\infty}$ the line rest wavelength and wind terminal speed. It is harder to determine $r$ though various methods are feasible with good data (Brown et al. 1994). However, the fact that $P$ maximises at a certain $r$ (Cassinelli et al. 1989; Brown et al. 1989) yields the relation (e.g., for the case $\theta \approx \pi / 2$ )

$$
N_{e}=8 \pi \sqrt{3} \frac{R^{2}}{\sigma_{T}} P_{\max }=6.5 \times 10^{44} R_{11}^{2} P_{-3}
$$

where $\sigma_{T}$ is the Thomson cross section, $R=10^{11} R_{11} \mathrm{~cm}$ is the photospheric radius, and $P_{-3}=10^{3} P_{\max }$.

Secondly, the total line luminosity $L$ of a blob for a collisionally dominated (e.g., recombination) line (like He II $\lambda .5411$ ) is given by $f(T) N_{e}^{2} / V$ where $f(T)$ is the line emissivity $\left(\mathrm{erg} \mathrm{cm}^{3} \mathrm{~s}^{-1}\right)$ at temperature $T$. Assuming $f$ is known approximately, then $L$ gives the value of $n^{2} V$ while $P$ gives $N_{\epsilon}=n V$ so that $n$ (and $V$ ) can be found, viz. (for $\theta \approx \pi / 2$ )

$$
n_{1}=\frac{\sigma_{T}}{8 \pi \sqrt{3} R^{2}} \frac{L}{f P_{\max }}=1.5 \times 10^{14} \mathrm{~cm}^{-3} \frac{L_{33}}{R_{11}^{2} f_{-26} P_{-3}}
$$

where $L_{33}=10^{-33} L$ and $f_{-26}=10^{26} f$.

Another estimate of $n$ can be obtained from Eq. (1) if we can estimate the blob linear size $l$ from the narrow feature spectral width $\Delta \lambda$. This depends on assumptions about the blob physics. Neglecting thermal ion motion and electron scattering within the blob for a supersonic optically thin blob, the simplest assumption concerning broadening is that the blob ions are moving ballistically with constant blob angular size $\Delta \theta$ (Brown et al. 1994). Con- 
sidering again blobs near $\theta=\pi / 2$ the broadening in this case is given by the spread in $v \cos \theta$ across $\Delta \theta$, leading to

$$
\Delta \theta=\frac{c \Delta \lambda}{v_{\infty} \lambda_{0}}
$$

and $l=r \Delta \theta$ where $r \approx R$ where $P=P_{\max }$. Combination of the above leads to a second estimate of $n$, viz.

$$
n_{2} \approx \frac{N}{4 s^{3}}=\frac{2 \pi \sqrt{3}}{R \sigma_{T}} \frac{P_{\max }}{\left(\Delta \lambda / \Delta \lambda_{\infty}\right)^{3}}=1.6 \times 10^{14} \mathrm{~cm}^{-3} \frac{P_{-3}}{R_{11}\left(\Delta \lambda / \Delta \lambda_{\infty}\right)^{3}}
$$

where $P_{-3}=P /(0.001)$. Results $n_{1}, n_{2}$ in Eqs. (2) and (4) are in remarkably good agreement and can be compared with the mean density $\bar{n}$ associated with the estimated mass loss rate $\dot{M}$ for a smooth spherical wind, namely $\bar{n} \approx 10^{13} \mathrm{~cm}^{-3} \dot{M}_{-5} / R_{11} v_{8}$ near the star for $\dot{M}=10^{-5} \dot{M}_{-5} M_{\odot} \mathrm{yr}^{-1}$ and wind speed $v=10^{8} v_{8} \mathrm{~cm} \mathrm{~s}^{-1}$. The fact that $n_{1}, n_{2} \gg \bar{n}$ shows that the wind must be fragmented (i.e., have a small filling factor) and is simply another way of saying that $\dot{M}$ can be reduced for a given total wind emission rate by fragmenting it (i.e., increasing $\int_{V} n^{2} d V$ for a given $\int_{V} n d V$ ).

\section{The blob distribution function}

In Section 3 we considered only single blobs. Some theories of blob origin also predict the distribution of blob parameters $N_{\epsilon}, l, n$, etc., in the wind as a whole, and correlations between them which are testable with data of the above sort, as discussed by Moffat et al. $(1992,1993)$ for their turbulence model according to which $n \propto l^{-1}$ so $N_{e} \propto l^{2}$ and $L \propto N_{e}^{1 / 2}$ while the blob size distribution is given by $d N / d N_{e} \propto N_{e}^{-\gamma}$ where $\gamma \approx 1-2$ in hierarchical turbulent systems. These properties are claimed to be in good agreement with observed data, but we are in the process of considering the rigour of the tests and uniqueness of the interpretation. For example our ballistic blob assumption leads to the relation $L \propto \Delta \lambda$ while the Moffat et al. (1993) model predicts $L \propto \Delta \lambda^{2}$. The latter is closer to the statistical trend in Robert's (1992) data but the former gave individual large blob densities in good agreement with an independent method. A second example where more work is needed is on relating $d N / d N_{e}$ to the observed photometric and polarimetric light curve variations, allowing for the distribution of blob sizes and locations, and the polarimetric cancellation of contributions from different azimuths. Preliminary analytic and numerical results (Richardson \& Brown 1994a,b) lend insight into why the polarimetric variability is much less than the photometric. For rather flat $d N / d N_{e}$ the variances turn out to be dominated by the few large blobs present and a ratio is predicted of $\sigma_{\text {phot }} / \sigma_{\text {pol }}=6 N^{1 / 2}$ where $N$ is the number of large blobs. The typical number of narrow features seen at one time is $N \approx 10$ giving a ratio of $\sigma_{\text {phot }} / \sigma_{\text {pol }} \approx 20$, very close to that observed. 


\section{The origin of the blobs}

An important question is whether WR wind blobs arise by non-uniform mass loss at the stellar surface or as a result of instability processes in the wind, as advocated by Moffat et al. (1993). A strong argument in favour of the former is given in Brown et al. (1994) on the basis of the polarimetry transients. After an initial increase at $r \approx R$, the optically thin polarisation contribution of a blob depends only on $N_{e} / r^{2}$, and so can only decrease since $N_{e}$ is invariant under (e.g., turbulent) redistribution of $n$ within it. This fact seems contrary to the Moffat et al. (1993) result that blobs are NOT seen at small $r$ insofar as the hot inner wind is best probed by lines of high ionisation potential ions. Resolution of this contradiction may lie in large blob optical depths obscuring individual blob line emission in this inner region.

\section{Acknowledgements}

We gratefully acknowledge the financial support of the LOC, Glasgow University, a Grant from the UK Science and Engineering Research Council, and an EEC Contract which enabled participation in the Symposium.

\section{References}

Brown, J.C., McLean, I.S. 1977, $A \& A$ 57, 141

Brown, J.C., McLean, I.S., Emslie, A.G. 1978, $A \& A 68415$

Brown, J.C., Carlaw, V.A., Cassinelli, J.P. 1989, ApJ 344, 341

Brown, J.C., Wood, K.H. 1991, A \& $A$ 265, 663

Brown, J.C., Richardson, L.L., Antohkin, I., Robert, C., Moffat, A.J.F., St-Louis, N. 1994, $A \mathscr{E} A$ submitted

Cassinelli, J.P., Nordsieck, K.H., Murison, M.A. 1987, ApJ 317, 290

Moffat, A.J.F., Robert, C. 1991, in: K.A. van der Hucht \& B. Hidayat (eds.), Wolf-Rayet Stars and Interrelations with other Massive Stars in Galaxies, Proc. IAU Symp. No. 143, (Dordrecht: Kluwer), p. 109

Owocki, S.P. 1994, in: A.F.J. Moffat \& N. St-Louis (eds.), Variability and Instability in Hot Star Winds, in press

Richardson, L.L., Brown, J.C. 1994a, these proceedings

Richardson, L.L., Brown, J.C. 1994b, in preparation

Robert, C. 1992, PhD thesis, University of Montréal

Wood, K.H., Brown, J.C., Fox, G.K. 1993, A\&A 271, 492 


\section{DISCUSSION:}

Israelian: What is the difference between small emission features in the flat-topped emission lines and discrete absorption components? Are they both due to blobs or shells?

Brown: Discrete absorption components can be due to spherical shells and also to blobs which happen to lie on the line of sight to the photospheric disk. The latter should be seen occasionally. Narrow emission features $\left(\Delta \lambda / \lambda_{0}<<v_{\infty} / \mathrm{c}\right)$ cannot be due to spherical shell emission because this produces all $\Delta \lambda / \lambda_{0} \approx\left(v_{\infty} / \mathrm{c}\right) \cos \theta$ between $\pm v \infty / \mathrm{c}$. A blob of 'width' $\Delta \theta$ $\ll 1$ produces observed flux only in $\Delta \lambda / \lambda o \approx \Delta \theta(\nu \infty / c)$.

Moffat: (1) In P Cyg lines we do see strong stochastic variations in the absorption edges, implying that we indeed are seeing blobs in strong absorption along the line of sight to the stellar disk.

(2) Lepine has now extended his wavelet analysis to emission lines of high ionization. They do indeed show blob structures, albeit at a relatively lower level (in units of the global line strength) than lines of low ionization. This is compatible with Robert's (1992, $\mathrm{PhD}$ thesis) calculations of the global variability of lines, showing that lines of higher ionization do vary, but at a lower relative level compared to low-ionization lines.

Brown: I am glad that your spectrometric data have now, contrary to your previous conclusions, proved consistent with the existence of blobs close to the star, as I have shown from the polarimetry. Thus both data sets support a subphotospheric rather than a wind origin for the blobs. 